\title{
Strategies To Enhance Transdermal Drug delivery
}

\author{
Mais Salim Saadallah*, Omar Abdulhakeem Hamid \\ Department of Pharmacology, College of Pharmacy, University of Mosul, Mosul, Iraq. \\ $\underline{\text { Received }} \underline{\text { Accepted }}$
}

\section{ABSTRACT}

Background: Transdermal drug delivery system (TDDS) is a promising delivery system that provides controlled drug release at a predetermined time. It has many advantages such as bypassing first-pass effect, increase patient convenience by providing single application rather than multiple dosing frequencies, and extends the action of short half-life drugs. There are three ways for drug penetration into the skin either via appendageal (shunt routes), intracellular route, and intercellular route. Permeation of drug substances through the stratum corneum remains a great challenge because it is the rate-limiting step for permeation for most molecules.

Aim: This review article highlights the advances and limitations of the strategies to enhance transdermal delivery of the drugs into the skin, including both physical and chemical methods.

Conclusion: The transdermal drug delivery system represents a good alternative for oral and parenteral therapy. Various penetration enhancing strategies have been successfully employed to enhance the permeation of low molecular weight molecules. For high molecular weight molecules, physical strategies such as microneedles can be used.

Keywords: transdermal drug delivery, permeation enhancement, nanocarriers, microneedles.

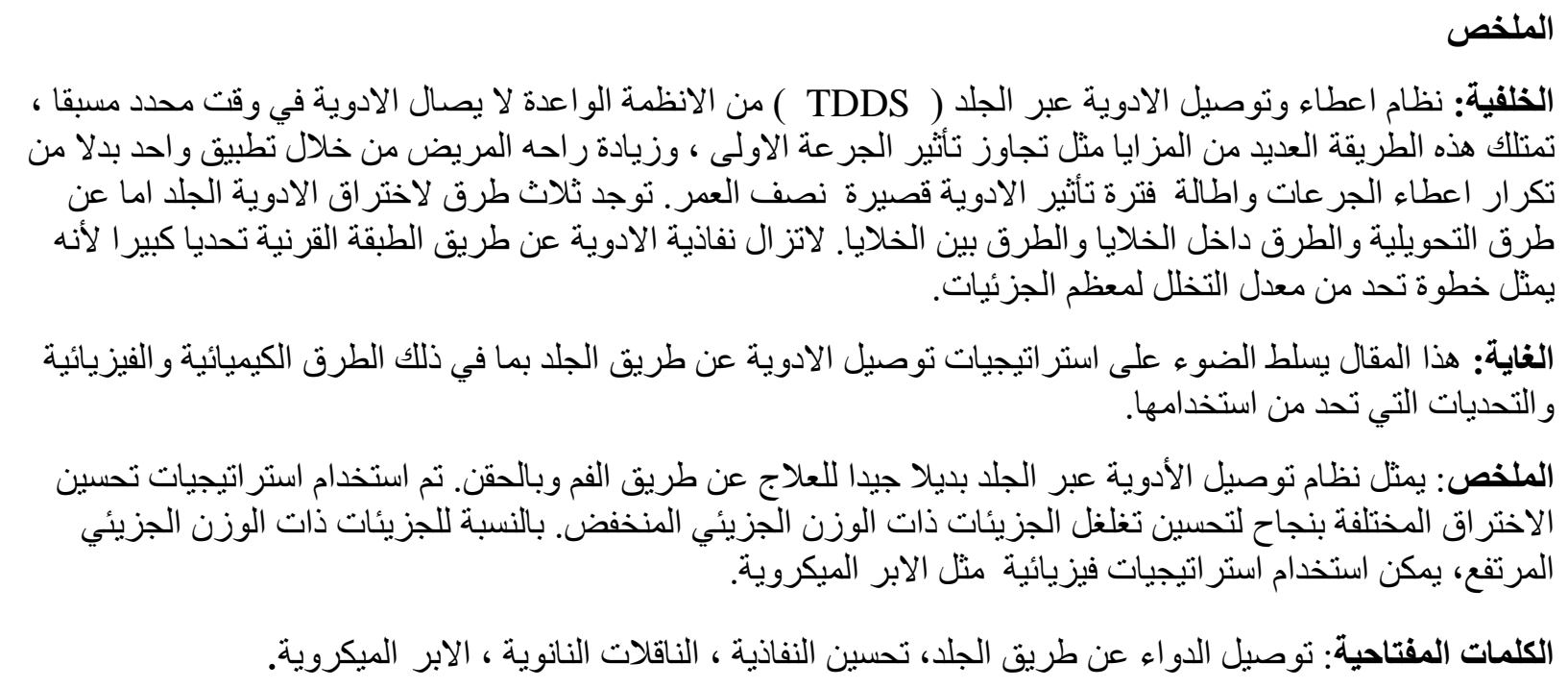

\section{Introduction}

$\mathrm{T}$ ransdermal drug delivery is the process of drug penetration via skin directly into the systemic circulation. Various dosage forms including patches or other transdermal forms applied to the skin such as cream, ointment, and gel (1). The skin is the organ that protects the whole body from external factors and is characterized by easy accessibility and large surface area (2). Dermal patches are the most common form of transdermal drug delivery (3). Patch application offers a 
painless way to deliver the drug and also bypassing the first-pass metabolism offering drug release in a controlled manner over an extended period of time. Transdermal patches vary in their design. In general, the patch consists of a backing layer that supports the patch, a drug layer which is in form of either a reservoir or a matrix, pressure -sensitive adhesive (PSA), and a release liner which is intended to be removed prior to patch application on the skin (4). Transdermal delivery systems have been studied for 50 years, and the first patch was approved by FDA in 1979 for the treatment of motion sickness that has contained scopolamine for three days of drug delivery. In addition to scopolamine, there are many other examples of drugs that are given as a transdermal patch such as nicotine to quit smoking, estrogen which is a hormone given in menopause and to prevent osteoporosis after menopause, nitroglycerin for angina, and lidocaine to relieve the pain of shingles (herpes zoster) (5). Nicotine patch was approved by the FDA in 1984 which after that helped over 1 million smokers gave up smoking (6). Unfortunately, not all drugs can be formulated as a transdermal patch; for a drug to penetrate the skin, should have molecular weight not exceeding a hundred Daltons and polar drugs cannot be delivered via the lipoid stratum corneum (7\&8).

\subsection{Advantages and Disadvantages of TDDS}

\subsubsection{Advantages of TDDS}

TDDS have several advantages over the oral route of drug administration. By administration of drug via the skin, gastrointestinal side effects of the drug such as bleeding, ulceration can be avoided. In addition, it provides a way to bypass the gastrointestinal first-pass effect, leading to enhanced drug bioavailability. Administration of drug via skin will also offer a way to extend the action of drugs that have a short half-life, wherein the patch provides a reservoir of the drug covered by a porous membrane to control the release of drug. Consequently, this will reduce dosing frequency and improve patient compliance. In the case of the patch application, drug action can be terminated easily by the removal of patch simply by the patient if side effects or toxicity occur (9).

\subsubsection{Disadvantages of TDDS}

Due to skin impermeability which is imposed by the stratum corneum, only potent drugs consider as suitable candidates for these systems. Application of TDDS may also cause different side effects such as contact dermatitis due to one or more components of the system and local irritation of the skin. Sometimes patch application may be uncomfortable and difficult to maintain its adherence for long periods $(10)(5)$.

\section{Skin Structure and Anatomy}

Human skin is considered as the largest organ of the body, comprising about $10 \%$ of the total body mass. It's a highly complex multilayered structure. For designing TDDS, the skin is considered a great challenge to formulations scientists. The properties of the skin limit the number of active ingredients that can be successfully delivered through the skin and achieve therapeutic 


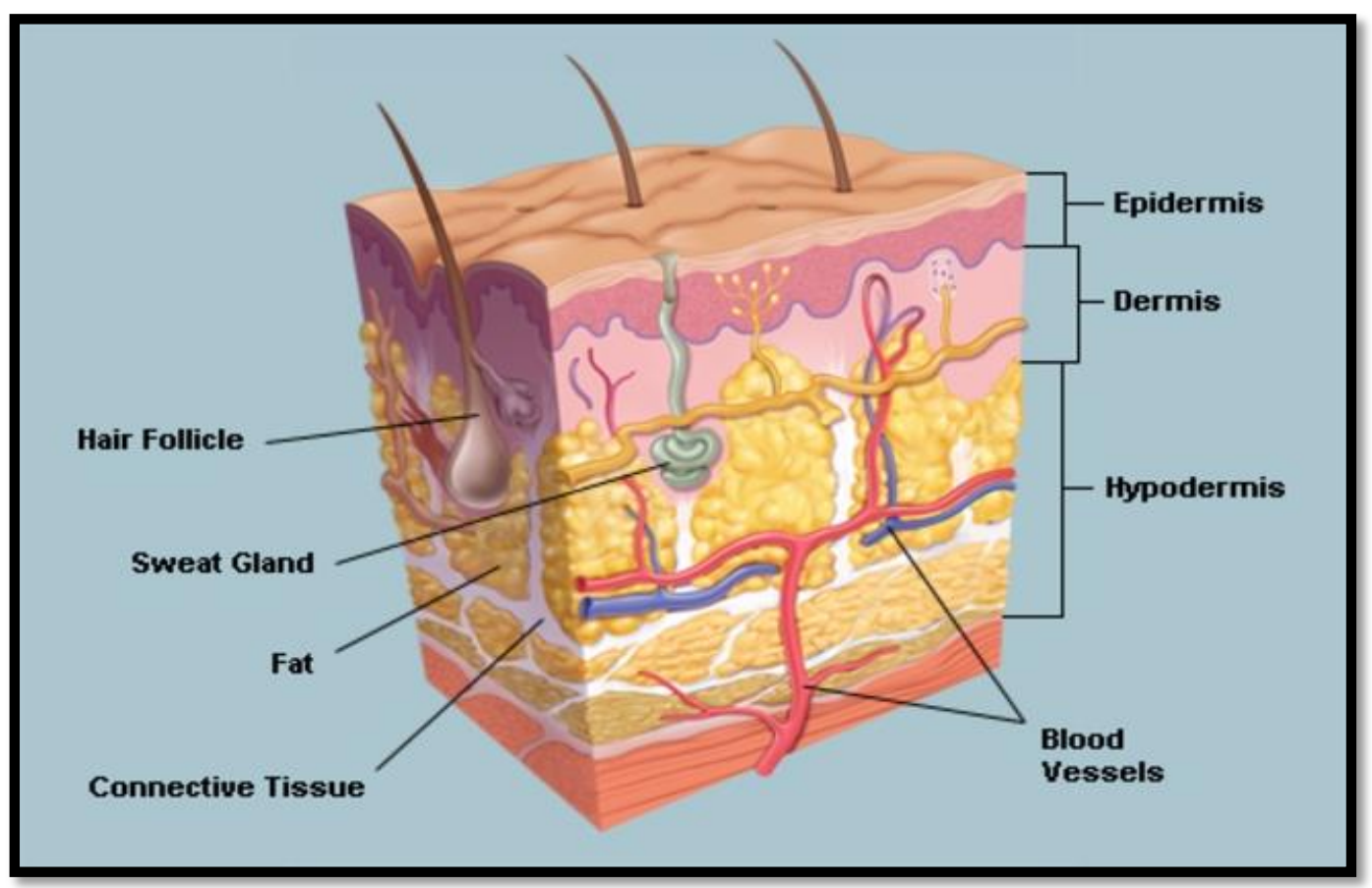

Figure 1: Human skin structure, this figure is adapted from Ref (72).

concentrations (11). Human skin as shown in figure (1) consists of three anatomically distinct tissues, epidermis, dermis, and hypodermis.

\subsection{Epidermis}

The epidermis is a stratified squamous epithelium layer. It consists of two types of cells: keratinocytes and dendritic cells. Keratinocytes are the main type of cells that synthesize the protein keratin. Desmosomes are protein bridges that connect the keratinocytes. The epidermis varies in thickness; ranging from $0.8 \mathrm{~mm}$ on the soles of the feet and palms of the hand down to $0.05 \mathrm{~mm}$ on the eyelids. Different stages of keratin maturation, as well as keratinocyte morphology and position give rise to four layers of the epidermis which are the stratum corneum (horny layer) and the remainder so-called viable epidermis that lies beneath the stratum corneum (12)(13).

Stratum corneum (SC) is the outermost layer of the skin; it is also called the horny layer which constitutes the main barrier for drug transfer as well as being a protective layer against invasion by foreign materials and prevents water loss. It is made up of layers of dead, hexagonal-shaped cornified cells called corneocytes. This layer varies in thickness according to its location in the skin, being the thickest on the palms and the soles. The barrier nature of this layer depends mainly on its constituents; the corneocytes are composed from proteins mainly, and are low in lipid content, surrounded by stacked layers of lipid bilayers. The epidermal transit time is about 28 days and it's known as the time that the epidermal cells take to move to this layer (12-14).

Viable epidermis it lies beneath the stratum corneum. It consists of various layers from 
the lower layers moving upwards include stratum basale (basal or germinativum cell layer) ,stratum spinosum (spinous or prickle cell layer), stratum granulosum (granular cell layer), stratum lucidum. The basal cell layer contains dividing and non- dividing keratinocytes, as these cells divide they move from this deep layer toward the surface, upon their movement they change both by morphology and histochemically, lose their nuclei and cytoplasm in the stratum granulosum layer and undergo keratinization to form the outermost layer of the stratum corneum $(15 \& 16)$.

\subsection{Dermis}

Dermis varies in thickness; ranging from 3 to $5 \mathrm{~mm}$ thick layer. It contains a matrix of connective tissue which includes blood vessels, lymph vessels, and nerves. Body temperature is regulated by continuous blood supply which also provides a continuous supply of oxygen and nutrients to the skin while removing toxins and waste products. Sink conditions for most penetrating molecules are maintained due to capillaries reach to within $0.2 \mathrm{~mm}$ of the skin surface. In turn, this keeps the dermal concentration of permeate low, and the resulting difference in concentration gradient across the epidermis is what provides the essential driving force for transdermal permeation (1).

\subsection{Hypodermis}

Hypodermis or alternatively subcutaneous fat layer. It represents a fat storage area that supports both the dermis and epidermis. This layer plays an important role in temperature regulation as well as nutritional and mechanical support. It carries principal blood vessels and nerves to skin and may contain sensory pressure organs. In the case of topical drug delivery, only penetration through the stratum corneum is essential and then retention of drug in skin layers is desired whereas, for transdermal drug delivery, the drug has to penetrate through all these three layers and reach into the systemic circulation (17-19).

\section{Drug Permeation Through the Skin}

There are three main ways by which drug molecules can cross the skin; via the appendageal (shunt routes), intracellular or transcellular route and intercellular route.

Skin appendages include sweat glands, and hair follicles with their associated sebaceous glands. They are of little importance since they compromise only $0.1 \%$ of the total skin area (20). Transcellular drug transport involves partitioning of drug molecules into keratin-filled corneocyte and then diffusing of the drug through the corneocyte before partitioning into the intercellular lipid region and then diffusing through it (11). In regard to the 'brick and mortar' structure of the stratum corneum, the intercellular route represents a great challenge for drug permeation. The drug molecule needs to pass through a tortuous pathway in contrast to the direct path that observed with the transcellular route. Another reason is that intercelullar route represents a region of alternating bilayers, as a result the drug molecule must partition into and diffuse through repeated aqueous and lipid domains. Most small unionized molecules can pass through this path (14). 


\section{Strategies to Enhance Transdermal Delivery}

Although transdermal delivery of the drugs seems to be a great alternative to other routes of drug delivery, permeation via the stratum corneum remains a big challenge. Stratum corneum is a formidable skin barrier and efforts have been made to overcome it. There are many ways to improve the transdermal permeation of drugs; they can be classified into active or physical and passive or chemical strategies.

\subsection{Physical Methods}

They involve the application of external stimuli for the active enhancement of transdermal permeation (21).

\subsubsection{Sonophoresis}

It refers to the application of ultrasound to enhance transdermal drug delivery. The exact mechanism of how ultrasound works to increase transdermal permeability is poorly understood. However, acoustic cavitation which structurally modifies stratum corneum lipids to create diffusional channels could be the possible mechanism of ultra-sound induced permeation(22). Increased skin permeation by thermal effects has been also hypothesized which increased skin permeability coefficient and drug diffusion coefficient (23). An enhancement of skin permeation by ultrasound widens the advantages and application of transdermal delivery. It can be classified into low, intermediate, and high-frequency sonophoresis according to the applied frequency. Sonophoresis can be used as pretreat for the skin before drug application as a means to increase its effect or as a formulation that can be applied at the same time. Although that there are many compounds are marketed as ultrasoundenhanced transdermal delivery such as Lidocaine which was approved in 2004, there are still some challenges regarding this technology which include the development of inexpensive ultrasound devices that can assure efficient transdermal delivery as well as safety of this technology remains an issue since high-frequency ultrasound may induce second-degree burns (21).

\subsubsection{Microneedles (MNs)}

Recently, MNs have been introduced as an effective way to enhance drug penetration into the skin. They are painless, micronized projections arranged in arrays, when applied to the skin it can increase percutaneous permeation dramatically. The first report on this system was in late 1990. Since stratum corneum thickness is only $10-20 \mu \mathrm{m}$, MNs with few hundreds of microns are capable of delivering the drug and bypass this barrier successfully (24). MNs are painless since they avoid the sensory nerve fibers (25). Structurally, MNs can be classified into four types namely, solid MNs, dissolving MNs, coated MNs, hollow MNs. Solid MNs can be used as skin pre-treatment to enhance percutaneous drug permeation. In this type, the MNs penetrated the skin, and then the drug is delivered by the channels that are generated, hence this type use the'poke and patch' approach of delivery. Various materials such as silicon, polymers including both biodegradable and non-degradable polymers such as polylactic-coglycolic acid (PLGA), maltose, and metals including stainless steel and nickel has been used to form solid MNs (26). Dissolving MNs are driving attention due to their advantages over the other types including high drug loading, convenience, and easy method of fabrication. This type uses the 'poke and release' approach of delivery which relies on the penetration of the skin by the microneedle patch and then drug release from the loaded dissolving tips. Polymers such as polyvinyl alcohol were utilized and dissolved four minutes after their insertion into the skin (27). Hollow MNs use the 'poke and flow' approach of delivery via 
micronized holes within the needles. Materials such as glass, silicone, and stainless steel can be used to fabricate this type of MNs. Flow rate via the holes has to be controlled since a high flow rate may cause pain. Besides drug delivery, hollow MNs can be exploited in blood extraction analysis as they utilize the poke and flow approaches. Coated MNs utilizes the 'coat and poke' approach of delivery, which includes insertion of the microneedle patch firstly into the skin followed by the release of the drug-coated on the microneedle tips. Coated MNs are solid MNs with superior advantage and patient compliance; they are considered as single-step administration systems comparing to solid MNs which are complex two-step administration systems(28). Stainless steel and titanium have been employed as materials to fabricate this system. In summary, MNs are advanced drug delivery systems that can be fabricated from a wide range of materials including polymers, inorganic materials, and metals with a variety of applications but safety concerns have to be considered such as pain associated with their administration and the potential of the materials used in fabrication to cause irritation and erythema and postapplication infection (29-31).

\subsubsection{Iontophoresis}

It involves the use of low current intensities $\left(0.5 \mathrm{~mA} / \mathrm{cm}^{2}\right)$ to enhance the transdermal permeation of ionized molecules. Phoresor $^{\mathrm{TM}}$ system was pioneered by IOMED inc. has been approved by the FDA as a physical medicine delivery device (32). Iontophoresis depends on the fact that like repels like; when ionized drug molecules are to be delivered, the positive molecules will be attracted to the electrode with the same polarity; anode, and when electromotive gradient passes it will go throw or into the stratum corneum (33). In the case of neutral or uncharged solutes, they can be candidates for iontophoresis as well after some modifications in their physicochemical characteristics such as synthesis of watersoluble prodrugs with ionizable moieties (34). There are three ways for drug permeation into the stratum corneum; transcellular and intercellular and trans appendageal route with the latter thought to be the least electromotive resistance (35). Many factors affecting iontophoresis drug delivery include the concentration of the drug molecule, $\mathrm{pH}$ of the system, current intensity, and permeant type (36). Drawbacks of iontophoresis include current limitations which is $0.5 \mathrm{~mA} / \mathrm{cm}^{2}$ or less and minor skin irritation and erythema at the site of application of iontophoretic patch (37).

\subsubsection{Thermal ablation}

Its a technique that involves the application of heat to increase drug permeation via the skin causing depletion of the SC without damaging the deeper underlying tissues with an exposure period ranging from 1 microsecond to 100 millisecond. The underlying mechanism involves stratum corneum vaporization followed by its removal without damaging the deeper tissues. This can be done in two ways: 1)moderate temperature (less than $100^{\circ} \mathrm{C}$ ) for a long period or2)high temperature (more than $100^{\circ} \mathrm{C}$ ) for short period with the latter being more favorable to avoid skin damage. Different ways can be used to perform thermal ablation include chemical heating, radiofrequency, and laser. Thermal ablation has been widely used to deliver macromolecules and vaccines but care should be taken since excessive heat may damage the deeper layers and cause thermohaemolysis(38\&39).

\subsubsection{Velocity based devices}

They include the application of velocity within the range of $100-200 \mathrm{~m} / \mathrm{s}$ to punctuate the skin making use of compressed gas or coiled spring as a power source. This technique was used mainly to deliver vaccines as well as macromolecules 
including monoclonal antibodies and hormones besides nucleic acids providing a needle-free approach based on jet injection for drug delivery. Although this technology offers a good alternative for the usage of conventional needles, it had some limitations reported during their usage including pain and bruising at the site of administration (40).

\subsection{Chemical Methods}

Chemical or passive methods to enhance transdermal permeation include: chemical penetration enhancers, eutectic systems, prodrug (41).

\subsubsection{Chemical enhancers}

Chemical enhancers are one of the most commonly used methods to enhance skin penetration. Drug partitioning into the skin can be increased by several mechanisms; either by extraction of intercellular lipids or proteins of the stratum corneum or by hydration of the stratum corneum which temporarily and reversibly change the barrier nature of the skin and increases drug penetration. Ideally, the chemical enhancer should be non-toxic and non-irritant, compatible with other ingredients, biodegradable, cheap, and available, and change the morphological nature of the skin reversibly. There are many classes of penetration enhancers depending on their chemical structure includes fatty alcohols like (propylene glycol, ethanol), fatty acids like (oleic acid), sulfoxides like ( dimethylsulfoxide DMSO and dimethylformamide DMF), terpenes like camphor, surfactants (cationic, anionic, zwitterion, non-ionic) and urea (42). The safety of penetration enhancers should be concerned since they have the ability to cause skin irritation including swelling, local inflammation, erythema, and dermatitis (43).

\subsubsection{Eutectic systems}

Eutectic systems are drug formulation that combines two compounds in an adequate ratio and the resultant mixture substance have a melting point lower than this of each compound alone. This lowering of melting point leads to an increment insolubility in the stratum corneum lipids and hence better skin penetration. EMLA® cream a topical local anesthetic is an example of a eutectic mixture that composed of lignocaine and prilocaine (1:1), when compared to a placebo, it dramatically reduced the pain associated with venous cannulation in children. However, the formulation of this system is challenging originated from the use of two functional components in one topical formulation (44\&45).

\subsubsection{Prodrug}

It's a compound that is inactive in its parent form and after entering the body it will convert back to its active form. This approach has been investigated for transdermal delivery; the resultant prodrug has different physicochemical properties including lipophilicity, partition coefficient and solubility, and as a result better skin penetration. Prodrug is formed upon modification of the chemical structure of the drug most commonly covalent linkage of the active drug with inactive moiety by an ester bond. Upon contact with skin, non-specific esterases of the skin will cleave the bond, liberating the active drug to exert its action (46\&47). Many drugs showed improved permeation making use of this approach for example diclofenac acid prodrugs formation to enhance transdermal delivery (47).

\subsection{Nanocarriers}

Various nanocarriers systems have been used to enhance transdermal delivery. These carriers can be divided into lipid-based formulations and polymeric nanoparticles. 


\subsubsection{Lipid-based formulations}

These include liposomes and their analogues and lipid nanoparticles.

\subsubsection{Liposomes}

They are spherical vesicular systems consist of one or more lipid bilayers that contain a hydrophobic head and a hydrophilic tail. They consist of phospholipids with or without cholesterol. Phosphatidylcholine is the main phospholipid from egg yolk or soybean. Their unique structure allows them to incorporate both hydrophilic and hydrophobic drugs besides being non-toxic and biodegradable systems(48). However, it has been reported that these systems are able only to stay at the skin surface without penetration into the deeper layers of the epidermis which eventually leading to minimal amounts of the loaded drug reaching the systemic circulation. As a result, these systems are mainly used for topical preparations to treat skin diseases providing prolonged retention of the drug with sustained-release(49).

Manca et al. incorporated allantoin that is used for skin scars treatment into conventional liposomes and an argan oil containing liposomes to increase skin hydration and improve local accumulation. For comparison, allantoin in water dispersion and commercial gel formulation (sameplast ${ }^{\circledR}$ gel) were used. In the case of conventional liposomes, allantoin accumulation in the dermis was double $\left(\sim 3.4 \mu \mathrm{g} / \mathrm{cm}^{2}, \mathrm{p}<0.05\right)$ with respect to that provided with the commercial gel. In argan oil liposomes, important drug accumulation improvement was observed in the epidermis $\left(\sim 2.2 \mu \mathrm{g} / \mathrm{cm}^{2} \mathrm{p}<0.05\right.$ versus all $)$ and mostly in the dermis $\left(\sim 8.7 \mu \mathrm{g} / \mathrm{cm}^{2} \mathrm{p}<0.01\right.$ versus all). Besides, both of conventional liposomes and argan oil liposomes showed a significant increase in the amount permeated via the skin which was $33 \mu \mathrm{g} / \mathrm{cm}^{2}(\mathrm{p}<0.05$ versus water dispersion and sameplast $\AA$ gel values) and only $17 \mu \mathrm{g} / \mathrm{cm}^{2}$ in case of water dispersion and sameplast ${ }^{\circledR} \operatorname{gel}(50)$.

\subsubsection{Niosomes}

Niosomes are liposome-like structures, they are vesicular systems made of non-ionic surfactants associated mainly with cholesterol. They lack phospholipids in their structure, therefore prevent oxidative degradation and provide higher chemical stability in comparison to liposomes (48). They have been widely investigated to enhance drug permeation in TDDS, for example A.S. Zidan et al. prepared simvastatin niosomal gels as a possible transdermal application system for pediatric. In-vivo pharmacokinetic data showed a significant reduction in cholesterol and triglycerides level with an increment in high-density lipoprotein level after transdermal niosomal administration to rats and an augmentation in simvastatin bioavailability by three folds approximately when compared to an oral drug suspension. This finding suggests that niosomes do not only have the ability to improve bioavailability and enhance hypolipidemic activity but also can be a promising dosage form to treat pediatric hyperlipidemia (51). Ethosomes are soft, flexible vesicular systems that contain ethanol in relatively high concentrations $20-45 \%$. They are composed of phospholipids bilayers enclosing the hydroalcoholic core. Hydrophilic and lipophilic molecules can be incorporated into ethosomes. The presence of ethanol in this system provides many advantages over other vesicular systems includes high permeation ability into the skin by acting as an effective penetration enhancer as well as the production of smaller particles when prepared under the same procedure besides, increases the flexibility and system fluidity(52). Ethosomes have a wide variety of applications includes microbial and viral 
skin infection treatment, testosterone ethosomes for hormonal deficiency and minoxidil ethosomes for hair loss, and other miscellaneous applications (52).

\subsubsection{Transfersomes}

Transfersomes have been identified in the 1990s by Cevc et al.(53). They are composed of phospholipid bilayers along with edge activator, water, and small percentage of alcohol. The presence of edge activator confers the deformability and ultraflexibility behavior of the transfersomes, providing an extraordinary ability for them to squeeze themselves into the barriers and pores of the skin and giving rise to excellent skin permeation(54). They are capable to squeeze themselves to about ten times smaller than vesicle diameter without deformation or fragmentation. Compared to liposomes, they can penetrate deeper into skin layers and reach the systemic circulation more efficiently. The most common edge activators used are surfactants such as span 80, tween 80, and sodium cholate. Besides vesicle flexibility, these surfactants can play a great role in skin permeation by fluidizing, and solubilizing skin lipids. Transfersomes have wide applications in transdermal delivery such as the delivery of antioxidants, anticancer drugs, corticosteroids, and antiinflammatory drugs (55).

\subsubsection{Invasomes}

They are vesicular nanocarriers that have been developed after the encouraging results of transfersomes. They consist of phospholipid bilayers, ethanol, and terpenes. The presence of terpenes act as penetration enhancers; they create elastic vesicles and fluidize stratum corneum lipids which make invasomes have a superior advantage over liposomes(56). Ethanol addition aids to create deformable vesicles as well by fluidizing the lipids in the vesicle structure and hence increasing their permeation. Invasomes are used to enhance the permeation of a wide variety of drugs examples including Avanafil, Isradipine, and phenyl ethyl resorcinol(57).

\section{Lipid nanoparticles}

They include solid-lipid nanoparticles, nanostructured lipid carriers, and nanoemulsions.

\subsubsection{Solid-lipid nanoparticles}

Solid-lipid nanoparticles are colloidal dispersions consist of solid, biodegradable lipid matrices, ranging in diameter (10-1000 $\mathrm{nm}$ ) and remain solid at room temperature (58). They can incorporate both lipophilic and hydrophilic drugs providing controlled release and excellent stability and protection of sensitive drugs from the surrounding environment. Besides, they can be used in targeted drug delivery (59). Major drawbacks of solid-lipid nanoparticles include limited drug loading and drug expulsion that follows the polymorphic transition of the solid lipids. Examples of drugs prepared as solid-lipid nanoparticles for cutaneous use include methotrexate and etanercept as combination therapy for topical psoriasis treatment and solid-lipid nanoparticles of aconitine which were prepared to improve drugs safety and permeation (60).

\subsubsection{Nanostructured lipid carriers}

The updated form of the solid-lipid nanoparticles. They were developed to overcome the limitations of solid-lipid nanoparticles. The internal core consists of solid lipid and liquid lipid which differs from solid-lipid nanoparticles wherein only solid lipids are present. They form a unique structure with high drug encapsulation efficiency and lesser drug discharge upon storage which makes them a suitable dosage form for long- term skin administration. When nanostructured lipid carriers have been used in topical delivery, they showed better skin targeting and permeation and minor skin irritation for many therapeutic agents such as flurbiprofen and 
indomethacin as anti-inflammatory agents and podophyllotoxin for warts treatment (61).

\subsubsection{Nanoemulsion}

Nanoemulsion is an emulsion with a very fine droplet size $(20-200 \mathrm{~nm})$ which is thermodynamically and kinetically stable (they do not show flocculation or coalescence upon storage). It consists of droplet (oil)phase dispersed in an aqueous phase and stabilized by surfactants or cosurfactants. Nanoemulsion is a lipid-based formulation and therefore IT is suitable for the incorporation of lipophilic compounds, improving their solubility and bioavailability (62). Drawbacks of this system may include a costly manufacturing process, the availability of surfactants and Ostwald ripening which includes the growth of larger droplets when the smaller droplets dissolve, and deposit on the larger ones(63). Examples of compounds delivered transdermally using NEs include clobetasol propionate and calcipotriol as nanoemulsion loaded gel for psoriasis treatment(64), and nanoemulsion of caffeine for transdermal delivery(65).

\subsubsection{Polymeric nanoparticles}

Polymeric nanoparticles are colloidal nanocarriers with a size range from 10 to $1000 \mathrm{~nm}$. A polymeric nanoparticle is composed of a polymer that contains a drug either encapsulated, adsorbed, or chemically linked to the surface of the particle. Their structure has been identified in 2002 by Discher and Eisenberg (66). Polymeric nanocarrier-enhanced topical delivery of drugs has obtained an interest since it offers several advantages compared to other nanocarriers such as protecting the encapsulated drug from degradation, providing sustained and controlled release, and hence reduces side effects especially of toxic drugs and increases skin permeation of the drugs as well as improving the bioavailability of poorly water soluble drugs
(67). Polymeric nanoparticles can be classified into two types, including nanospheres and nanocapsules according to their structure. Nanospheres or matrix systems consists of a matrix in which both the drug and the polymer are uniformly dispersed, whereas nanocapsules have a core-shell structure with the active compound being encapsulated in an aqueous or oily core and surrounded by the polymer around (66). Their relatively small size, and surface modifications of polymeric nanoparticles can greatly enhance the interaction of the active substance with their targeted site (68).

P. Dong et al. investigated the cutaneous penetration of model drugs using $\mathrm{pH}^{-}$ sensitive Eudragit L100 polymeric nanoparticles. In the case of dexamethasoneloaded Eudragit L100 PNPs, results showed an improvement in cutaneous penetration in comparison to a commercial cream. Besides, in-vitro rapid release of the drug was triggered by $\mathrm{pH}$ above than 5.9, and that faster drug release and higher penetration into skin layer obtained in barrier-disrupted skin. For the other drug, which is lipophilic, Nile red loaded with Eudragit L100 PNPs showed high transfollicular penetration of the drug and improved cutaneous penetration and controlled drug release of this lipophilic drug and most favorably on barrier-disrupted skin (69). In a different study, dexamethasone-loaded PNPs were formulated using various polymers, including Eudragit L100. The results showed that Eudragit polymers including the Eudragit L100 were dissolved at $\mathrm{pH} 7.5$ and released $65-70 \%$ of the drug within 7 hours whereas they remained intact at $\mathrm{pH} 6$ buffers and released the drug slowly and this ensures the $\mathrm{pH}$-dependant action of Eudragit L100 and that it releases the drug in a different manner at the skin surface whereas it dissolves and gives fast release at the deeper hair follicles as it has higher $\mathrm{pH}$ levels (70). 
Another study reported by Rajesh et al. included the preparation of Curcuminencapsulated Chitosan Nanoparticles for transdermal delivery. In-vitro release studies for the nanoparticles were performed at two different $\mathrm{pH}$; acetate buffer $\mathrm{pH} 5$ and PBS $\mathrm{pH} \quad 7.4$ to simulate skin $\mathrm{pH}$ and physiological $\mathrm{pH}$, respectively. Drug release results showed that no initial burst release was seen at either $\mathrm{pH}$ with the controlled release over time. At $\mathrm{pH} 5,41.5 \pm 1.1 \%$ of the drug released by the end of 24 hours whereas only $19 \%$ of the drug was released in PBS 7.4 at the same period due to $\mathrm{pH}$ dependant swelling behavior of the polymer, Chitosan. In-vitro permeation results using the Strat-M® membrane showed that a significant amount of Curcumin permeated from both formulations (3:1 and 5:1) in comparison to control (curcumin solution) $(\mathrm{p}<0.05)(71)$.

\section{References}

1. Mali AD. An updated review on transdermal drug delivery systems. Skin (Los Angeles). 2015;8(9).

2. Münch S, Wohlrab J, Neubert RHH. Dermal and transdermal delivery of pharmaceutically relevant macromolecules. Eur J Pharm Biopharm. 2017;119:235-42.

3. Transdermal Drug Delivery [Internet]. [cited 2020 Dec 27]. Available from: https://studylib.net/doc/8884310/trans dermal-drug-delivery

4. Shargel L, Yu ABC. Applied Biopharmaceutics \& Pharmacokinetics. Seventh. McGrawHill Education; 2016. 910 p.

5. Lewis S, Pandey S, Udupa N. Design and evaluation of matrix type and membrane controlled transdermal delivery systems of nicotine suitable for use in smoking cessation. Indian J

\section{Conclusion}

The transdermal drug delivery system represents a good alternative for oral and parenteral therapy. Various penetration enhancing strategies have been successfully employed to enhance the permeation of low molecular weight molecules. For high molecular weight molecules, active strategies such as microneedles and iontophoresis can be used. Nanotechnology has derived great attention lately; by fabricating any kind of drugs into compounds within the nanometer range, permeation into skin could be enhanced greatly but challenges remain in the number of manufacturing steps as well as the cost of characterization methods.

Pharm Sci. 2006;68(2):179-84.

6. Pastore MN, Kalia YN, Horstmann M, Roberts MS. Transdermal patches: History, development and pharmacology. $\mathrm{Br} \quad \mathrm{J}$ Pharmacol. 2015;172(9):2179-209.

7. Prausnitz MR, Langer R. Nihms121685. Nat Biotechnol. 2009;26(11):1261-8.

8. Al Hanbali OA, Khan HMS, Sarfraz M, Arafat M, Ijaz S, Hameed A. Transdermal patches: Design and current approaches to painless drug delivery. Acta Pharm. 2019;69(2):197-215.

9. Kriplani , p., Sharma , A., Pun, P., chopra, B., Dhingra, A. and Deswal G. Formulation and evaluation of transdermal patch of diclofenac sodium. Glob J Pharm Pharm Sci. 2018;4(5):1-4.

10. Escobar-Chávez JJ, Díaz-Torres R, Rodríguez-Cruz IM, DomínguezDelgado CL, Morales RS, ÁngelesAnguiano E, et al. Nanocarriers for 
transdermal drug delivery. Res Reports Transdermal Drug Deliv. 2012;1:3-17.

11. Aulton ME, Taylor K. Aulton's Pharmaceutics: The Design and Manufacture of Medicines. 2013: Churchill Livingstone. Elsevier;

12. Venus M, Waterman J, McNab I. Basic physiology of the skin. Surg. 2010;28(10):469-72.

13. Kolarsick PAJ, Kolarsick MA, Goodwin C. Anatomy and physiology of the skin. J Dermatol Nurses Assoc. 2011;3(4):203-13.

14. Rastogi V, Yadav P. Transdermal drug delivery system: An overview. Asian J Pharm Free full text Artic from Asian J Pharm. 2014;6(3).

15. Malvey S, Rao JV, Arumugam KM. Transdermal drug delivery system: A mini review. Pharma Innov. 2019;8:181-97.

16. Sharma N, Agarwal G, Rana AC, Bhat ZA, Kumar D. A review: transdermal drug delivery system: a tool for novel drug delivery system. Int J drug dev Res. 2011;3(3):70-84.

17. Gaikwad AK. Transdermal drug delivery system: Formulation aspects and evaluation. Compr J Pharm Sci. 2013;1(1):1-10.

18. Shankar PB, Manohar SD, Bhanudas SR, Anjaneri N. Patches: A Novel approach for development of topical drug delivery system. J Adv Pharm Educ Res Oct-Dec. 2013;3(4).

19. Kesarwani A, Yadav AK, Singh S, Gautam H, Singh HN, Sharma A, et al. Theoretical aspects of transdermal drug delivery system. Bull Pharm Res. 2013;3(2):78-89.

20. Sonkar R, Prajapati SK, Chanchal DK, Bijauliya RK, Kumar S. A review on transdermal patches as a novel drug delivery system. Int $\mathbf{J}$ Life Sci Rev. 2018;4(4):52-62.

21. Szunerits S, Boukherroub R. Heat: a highly efficient skin enhancer for transdermal drug delivery. Front Bioeng Biotechnol. 2018;6:15.

22. Seah BC-Q, Teo BM. Recent advances in ultrasound-based transdermal drug delivery. Int $\mathbf{J}$ Nanomedicine. 2018;13:7749.

23. Ita K. Recent progress in transdermal sonophoresis. Pharm Dev Technol. 2017;22(4):458-66.

24. Henry S, McAllister D V, Allen MG, Prausnitz MR. Microfabricated microneedles: a novel approach to transdermal drug delivery. J Pharm Sci. 1998;87(8):922-5.

25. Kim Y-C, Park J-H, Prausnitz MR. Microneedles for drug and vaccine delivery. Adv Drug Deliv Rev. 2012;64(14):1547-68.

26. Larraneta E, Lutton REM, Woolfson AD, Donnelly RF. Microneedle arrays as transdermal and intradermal drug delivery systems: Materials science, manufacture and commercial development. Mater Sci Eng R Reports. 2016;104:1-32.

27. Yang H, Ye L, Guo XD, Yang C, Compans RW, Prausnitz MR. Ebola vaccination using a DNA vaccine coated on PLGA-PLL $/ \gamma$ PGA nanoparticles administered using a microneedle patch. Adv Healthc Mater. 2017;6(1):1600750.

28. Tuan-Mahmood T-M, McCrudden MTC, Torrisi BM, McAlister E, Garland MJ, Singh TRR, et al. Microneedles for intradermal and transdermal drug delivery. Eur J Pharm Sci. 2013;50(5):623-37.

29. Hao Y, Li W, Zhou X, Yang F, Qian Z. Microneedles-based transdermal drug delivery systems: a review. J Biomed Nanotechnol. 2017;13(12):1581-97.

30. Dharadhar S, Majumdar A, Dhoble S, Patravale V. Microneedles for transdermal drug delivery: a 
systematic review. Drug Dev Ind Pharm. 2019;45(2):188-201.

31. Queiroz MLB, Shanmugam S, Santos LNS, Campos C de A, Santos AM, Batista MS, et al. Microneedles as an alternative technology for transdermal drug delivery systems: a patent review. Expert Opin Ther Pat. 2020;30(6):433-52.

32. Mathur V, Satrawala Y, Rajput MS. Physical and chemical penetration enhancers in transdermal drug delivery system. Asian J Pharm Free full text Artic from Asian J Pharm. 2014;4(3).

33. Bavaskar K, Jain A, Patil M, Kalamkar R. The impact of penetration enhancers on transdermal drug delivery system: physical and chemical approach. Int $\mathbf{J}$ Phar Res Rev. 2015;4(7):14-24.

34. Chen Y, Alberti I, Kalia YN. Topical iontophoretic delivery of ionizable, biolabile aciclovir prodrugs: A rational approach to improve cutaneous bioavailability. Eur J Pharm Biopharm. 2016;99:103-13.

35. Sheikh NK, Dua A. Iontophoresis Analgesic Medications. StatPearls [Internet]. 2020;

36. Kaur T. Transdermal drug delivery by iontophoresis: Mechanistic aspects.

37. Bakshi P, Vora D, Hemmady K, Banga AK. Iontophoretic skin delivery systems: Success and failures. Int J Pharm. 2020;119584.

38. Lee JW, Gadiraju P, Park J-H, Allen MG, Prausnitz MR. Microsecond thermal ablation of skin for transdermal drug delivery. J Control release. 2011;154(1):58-68.

39. Parhi R, Mandru A. Enhancement of skin permeability with thermal ablation techniques: concept to commercial products. Drug Deliv Transl Res. 2020;1-25.

40. Hogan NC, Taberner AJ, Jones LA,
Hunter IW. Needle-free delivery of macromolecules through the skin using controllable jet injectors. Expert Opin Drug Deliv. 2015;12(10):163748.

41. Alkilani AZ, McCrudden MTC, Donnelly RF. Transdermal drug delivery: innovative pharmaceutical developments based on disruption of the barrier properties of the stratum corneum. Pharmaceutics. 2015;7(4):438-70.

42. Kapoor A, Mishra SK, Verma DK, Pandey P. Chemical penetration enhancers for transdermal drug delivery system. J Drug Deliv Ther. 2018;8(5-s):62-6.

43. Karande P, Mitragotri S. Enhancement of transdermal drug delivery via synergistic action of chemicals. Biochim Biophys Acta (BBA)-Biomembranes. 2009; 1788(11):2362-73.

44. Jones SA, Fiala S, Brown MB. Eutectic Systems for Penetration Enhancement. In: Dragicevic N, Maibach HI, editors. Percutaneous Penetration Enhancers Chemical Methods in Penetration Enhancement: Drug Manipulation Strategies and Vehicle Effects [Internet]. Berlin, Heidelberg: Springer Berlin Heidelberg; 2015. p. 163-73. Available from: https://doi.org/10.1007/978-3-66245013-0_12

45. Atkinson JP, Maibach HI, Dragicevic $\mathrm{N}$. Targets in Dermal and Transdermal Delivery and Classification of Penetration Enhancement Methods. In: Dragicevic N, Maibach HI, editors. Percutaneous Penetration Enhancers Chemical Methods in Penetration Enhancement: Drug Manipulation Strategies and Vehicle Effects [Internet]. Berlin, Heidelberg: 
Springer Berlin Heidelberg; 2015. p. 93-108. Available from: https://doi.org/10.1007/978-3-66245013-0_8

46. Anantrao JH, Nath PA, Nivrutti PR. Drug Penetration Enhancement Techniques in Transdermal Drug Delivery System: A Review. J Pharm Res Int. 2021;46-61.

47. Lobo S, Li H, Farhan N, Yan G. Evaluation of diclofenac prodrugs for enhancing transdermal delivery. Drug Dev Ind Pharm. 2014;40(3):425-32.

48. Jijie R, Barras A, Boukherroub R, Szunerits S. Nanomaterials for transdermal drug delivery: beyond the state of the art of liposomal structures. J Mater Chem B. 2017;5(44):8653-75.

49. Vanić Ž, Hafner A, Bego M, ŠkalkoBasnet N. Characterization of various deformable liposomes with metronidazole. Drug Dev Ind Pharm. 2013;39(3):481-8.

50. Manca ML, Matricardi P, Cencetti C, Peris JE, Melis V, Carbone C, et al. Combination of argan oil and phospholipids for the development of an effective liposome-like formulation able to improve skin hydration and allantoin dermal delivery. Int J Pharm. 2016;505(12):204-11.

51. Zidan AS, Hosny KM, Ahmed OAA, Fahmy UA. Assessment of simvastatin niosomes for pediatric transdermal drug delivery. Drug Deliv. 2016;23(5):1536-49.

52. Nainwal N, Jawla S, Singh R, Saharan VA. Transdermal applications of ethosomes-a detailed review. J Liposome Res. 2019;29(2):103-13.

53. Rai S, Pandey V, Rai G. Transfersomes as versatile and flexible nano-vesicular carriers in skin cancer therapy: the state of the art. Nano Rev Exp. 2017;8(1):1325708.

54. Li J, Wang $\mathrm{X}$, Zhang $\mathrm{T}$, Wang $\mathrm{C}$, Huang $Z$, Luo $X$, et al. A review on phospholipids and their main applications in drug delivery systems. Asian J Pharm Sci. 2015;10(2):81-98.

55. Opatha SAT, Titapiwatanakun V, Chutoprapat R. Transfersomes: A promising nanoencapsulation technique for transdermal drug delivery. Pharmaceutics. 2020;12(9):855.

56. Jain S, Tripathi S, Tripathi PK. Invasomes: Potential vesicular systems for transdermal delivery of drug molecules. J Drug Deliv Sci Technol. 2020;102166.

57. Babaie S, Bakhshayesh AR Del, Ha JW, Hamishehkar H, Kim KH. Invasome: A novel nanocarrier for transdermal drug delivery. Nanomaterials. 2020;10(2):341.

58. Winter E, Pizzol CD, Locatelli C, Crezkynski-Pasa TB. Development and evaluation of lipid nanoparticles for drug delivery: Study of toxicity in vitro and in vivo. $\mathrm{J}$ Nanosci Nanotechnol. 2016;16(2):1321-30.

59. Paliwal R, Paliwal SR, Kenwat R, Kurmi B Das, Sahu MK. Solid lipid nanoparticles: a review on recent perspectives and patents. Expert Opin Ther Pat. 2020;30(3):179-94.

60. Zhou X, Hao Y, Yuan L, Pradhan S, Shrestha K, Pradhan O, et al. Nanoformulations for transdermal drug delivery: a review. Chinese Chem Lett. 2018;29(12):1713-24.

61. Khosa A, Reddi S, Saha RN. Nanostructured lipid carriers for sitespecific drug delivery. Biomed Pharmacother. 2018;103:598-613.

62. Kaci M, Belhaffef A, Meziane S, Dostert G, Menu P, Velot E, et al. Nanoemulsions and topical creams for the safe and effective delivery of 
lipophilic antioxidant coenzyme Q10. Colloids Surfaces B Biointerfaces. 2018;167:165-75.

63. Naseema A, Kovooru L, Behera AK, Kumar KPP, Srivastava P. A critical review of synthesis procedures, applications and future potential of nanoemulsions. Adv Colloid Interface Sci. 2020;102318.

64. Kaur A, Katiyar SS, Kushwah V, Jain S. Nanoemulsion loaded gel for topical co-delivery of clobitasol propionate and calcipotriol in psoriasis. Nanomedicine Nanotechnology, Biol Med. 2017;13(4):1473-82.

65. Shakeel F, Ramadan W. Transdermal delivery of anticancer drug caffeine from water-in-oil nanoemulsions. Colloids Surfaces B Biointerfaces [Internet]. 2010;75(1):356-62. Available from: https://www.sciencedirect.com/scienc e/article/pii/S0927776509004202

66. Ahlawat J, Henriquez G, Narayan M. Enhancing the delivery of chemotherapeutics: role of biodegradable polymeric nanoparticles. Molecules. 2018;23(9):2157.

67. Garg U, Jain K. Dermal and Transdermal Drug Delivery through Vesicles and Particles: Preparation and Applications. Adv Pharm Bull. 2021;

68. Moritz M, Geszke-Moritz M. Recent developments in the application of polymeric nanoparticles as drug carriers. Adv Clin Exp Med. 2015;24(5):749-58.

69. Dong P, Sahle FF, Lohan SB, Saeidpour S, Albrecht S, Teutloff C, et al. pH-sensitive Eudragit ${ }^{\circledR}$ L 100 nanoparticles promote cutaneous penetration and drug release on the skin. J Control Release. 2019;295:214-22.

70. Sahle FF, Gerecke C, Kleuser B, Bodmeier R. Formulation and comparative in vitro evaluation of various dexamethasone-loaded $\mathrm{pH}$ sensitive polymeric nanoparticles intended for dermal applications. Int $\mathbf{J}$ Pharm. 2017;516(1-2):21-31.

71. Nair RS, Morris A, Billa N, Leong CO. An evaluation of curcuminencapsulated chitosan nanoparticles for transdermal delivery. Aaps Pharmscitech. 2019;20(2):1-13.

72. The Skin (Human Anatomy): Picture, Definition, Function, and Skin Conditions [Internet]. [cited 2021 Jan 21]. Available from: https://www.webmd.com/skinproblems-and-treatments/picture-ofthe-skin

73. Escobar-Chávez JJ, Rodríguez-Cruz IM, Domínguez-Delgado CL, DíazTorres R, Revilla-Vázquez AL, Aléncaster NC. Nanocarrier systems for transdermal drug delivery. Recent Adv Nov Drug Carr Syst. 2012; 